\title{
Multiple order parameter theory of surface melting: $A$ van der Waals approach
}

\author{
H. Löwen, T. Beier, and H. Wagner \\ Sektion Physik der Universität München, Federal Republic of Germany
}

Received October 30, 1989

We describe a van der Waals approach with multiple order parameter for surface melting near the triple point $T_{T}$ which is based on density functional theory for bulk freezing. The occurrence of surface-induced melting, nonmelting or blocked (incomplete) melting is governed by the specific form of the long-ranged tail in the interparticle potential. In the case of melting, the residual crystallinity at the vapor side of the quasi-liquid layer is shown to decay with a stretched exponential (power) law in $T_{T}-T$ for long-(short-) ranged interparticle forces

\section{Introduction}

At the surface of a solid the crystalline order is broken and the atoms are less tightly bound than in the bulk of the material. Hence, when a crystal is heated up along the sublimation line, it is likely that melting commences at the surface already below the bulk triple point temperature $T_{T}$ and proceeds via a growing quasi-liquid layer, which wets the solid-vapor interface as the temperature $T$ is raised towards $T_{T}$.

Recent ion-scattering [1-4] and LEED [5] experiments on metals and specific heat measurements of multilayer rare gas films [6] actualize and strongly support this ancient idea [7] of a surface induced premelting [8].

On the theoretical side a number of attempts have been made to deal with surface melting; these include, for instance: classical phonon theory [9], a mean-field lattice model [10], phenomenological Landau theories [11-13], a Kosterlitz-Thouless-like approach [14] and molecular dynamics simulations of both rare gas crystals $[15-18]$ and metals $[19,20]$. Despite these efforts, it must be admitted that the current understanding of the microscopic aspects of surface melting is still rather incomplete.

A major problem arises from the fact that one has to cope with a strongly inhomogeneous system involving density variations on several distinct but still comparable length scales. This feature is illustrated in Fig. 1 which shows a laterally averaged density profile in the direction $z$, perpendicular to the solid-vapor interface of a Lennard-Jones crystal. Apart from the lattice distance, the discernible additional length scales are the width of the intervening liquid layer and the widths of the transition regions layer/crystal and layer/vapor. We note that the decay of the crystalline order extends over several lattice distances in a relatively smooth manner as compared to the rapid lattice periodicity.

In this paper we propose a van der Waals theory of surface melting based on the density functional method. In order to motivate the crucial approximation underlying our approach, we write the local density of a

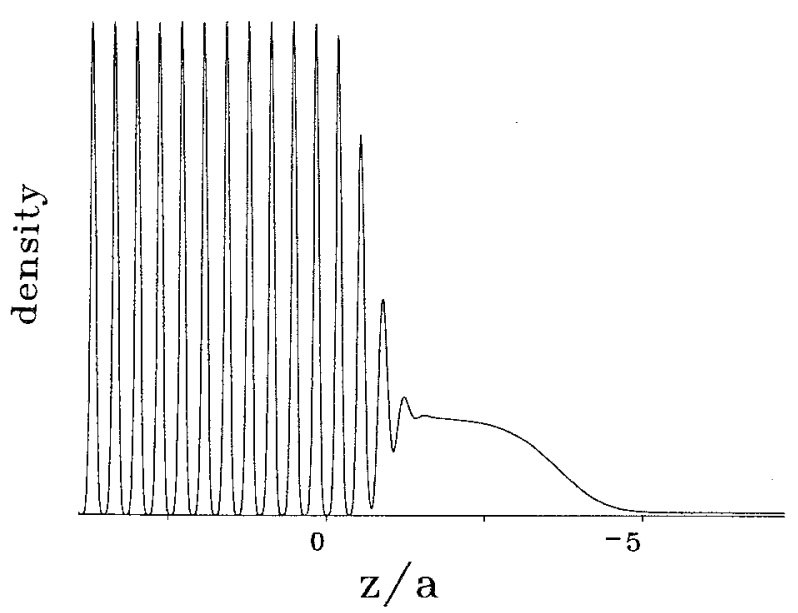

Fig. 1. Laterally averaged density in (110) direction of an fec-Lennard-Jones crystal with lattice constant $a$. The figure displays a well developed wetting layer between the crystal phase on the left and the vapor phase on the right at a reduced temperature $t$ $=1.5 \cdot 10^{-4}$. This density profile was obtained from the present van der Waals theory combined with the weighted density approximation for the free energy 
crystal as a Fourier series

$\rho(\mathbf{r})=\sum_{\mathbf{G}} \rho_{\mathbf{G}} \exp (\mathbf{i} \mathbf{G} \cdot \mathbf{r})$,

with the summation running over the reciprocal lattice $\{\mathbf{G}\}$. The Fourier coefficients $\left\{\rho_{\mathbf{G}}\right\}$ constitute a set of temperature-dependent but spatially constant order parameters (including the mean density for $\mathbf{G}=0$ ) for the interior bulk crystal which occupies the half-space $z>0$. However, in the interfacial region near $z=0$, these order parameters will be spatially modulated, $\rho_{\mathbf{G}}=b_{\mathbf{G}}(z)$, as indicated in Fig. 1. Our assumption is now that the coefficients $b_{\mathbf{G}}(z)$ vary smoothly over lattice distances so that a gradient expansion in the free energy functional may be employed.

An important advantage of this procedure as compared to phenomenological Landau models [11-13] is that the required microscopic input data are specified and practically calculable from the thermostatistics of bulk freezing, which has advanced considerably in the last few years [21].

A brief account of our work has been given previously [22]. Here, we supply a more detailed discussion of analytic results obtained from simplified free energy functionals, where attention is focused on the effect of long-ranged atomic forces upon the growth of the wetting layer and the residual crystallinity at the disordered surface.

\section{Van der Waals theory}

\section{Density functional approach}

We start from the grand canonical free energy functional $\Omega[\rho]$ for an inhomogeneous system with local density $\rho(\mathbf{r})$, temperature $\beta^{-1}$, chemical potential $\mu$ and volume $V$. Usually, $\Omega[\rho]$ is constructed as a sum of three terms [23]:

$\Omega[\rho]=F_{i d}[\rho]+F_{\text {exc }}[\rho]-\mu \int_{V} \mathrm{~d}^{3} r \rho(\mathbf{r})$,

where

$F_{i d}[\rho]=\beta^{-1} \int_{V} \mathrm{~d}^{3} r \rho(\mathbf{r})\left[\ln \left(A^{3} \rho(\mathbf{r})\right)-1\right]$,

is the local ideal gas contribution; $\dot{A}$ denotes the thermal wavelength of the particles and $F_{\mathrm{exc}}[\rho]$ is the excess Helmholtz functional.

In order to write down $F_{\text {exc }}[\rho]$ more explicitly, we assume a pairwise interparticle potential $\Phi(r)$ which is split into a short-ranged part $\Phi_{s}(r)$ and a long-ranged tail $\Phi_{l}(r)$ (see Fig. 2):

$\Phi(r)=\Phi_{s}(r)+\Phi_{l}(r)$.

$\Phi_{s}(r)$ includes both a repulsive core and a short-ranged attractive part. With $\Phi_{l}(r)$ treated in meanfield fashion,
$F_{\text {exc }}[\rho]$ is given by

$F_{\text {exc }}[\rho]=F_{s}[\rho]+(1 / 2) \iint_{V} \mathrm{~d}^{3} r \mathrm{~d}^{3} r^{\prime} \Phi_{l}\left(\left|\mathbf{r}-\mathbf{r}^{\prime}\right|\right) \rho(\mathbf{r}) \rho\left(\mathbf{r}^{\prime}\right)$.

Finally, $F_{s}[\rho]$ may be written in the compact form [23]

$$
\begin{aligned}
F_{s}[\rho]= & \beta^{-1} \int_{0}^{1} \mathrm{~d} \alpha(\alpha-1) \\
& \cdot \iint_{V} \mathrm{~d}^{3} r \mathrm{~d}^{3} r^{\prime} c_{s}\left(\mathbf{r}, \mathbf{r}^{\prime} ;[\alpha \rho]\right) \rho(\mathbf{r}) \rho\left(\mathbf{r}^{\prime}\right),
\end{aligned}
$$

where $c_{s}\left(\mathbf{r}, \mathbf{r}^{\prime} ;[\rho]\right)$ denotes the direct correlation function (DCF) arising from the short-ranged potential $\Phi_{s}(r)$. The usual procedure is to replace the repulsive part of $\Phi_{s}(r)$ by a hard core with diameter $\sigma$ and to treat the attractive part of $\Phi_{s}(r)$ perturbatively [24], so that

$c_{s}\left(\mathbf{r}, \mathbf{r}^{\prime} ;[\rho]\right) \cong c_{\mathrm{HS}}\left(\mathbf{r}, \mathbf{r}^{\prime} ;[\rho]\right)-\beta \widehat{\Phi}_{s}\left(\left|\mathbf{r}-\mathbf{r}^{\prime}\right|\right)$.

Here, the first term, $c_{\mathrm{HS}}$, is the DCF of a hard-core system, which may be constructed from the weighted density approximation [25]. $\hat{\Phi}_{s}(r)$ is the WCA-modification [24] of the attractive part of $\Phi_{s}(r)$, whereby short-distance effects are taken into account approximately.

The free energy functional (2.1) with these approximations yields a qualitatively realistic bulk phase diagram with a sublimation line and a triple point [26] and should also be a suitable basis for the study of interfacial structures at phase coexistence.

By (2.3)-(2.4), $\Omega[\rho]$ is separated into a short-ranged part $\Omega_{s}[\rho]$ and a long-ranged one, $F_{l}[\rho]$, which involves the meanfield term in (2.4). In what follows, further approximations such as (2.6) are not required.

In (2.3), a natural choice of the separation length is $2 \sigma-3 \sigma$, see Fig. 2. We expect the results below to be insensitive to the ambiguity in the splitting procedure.

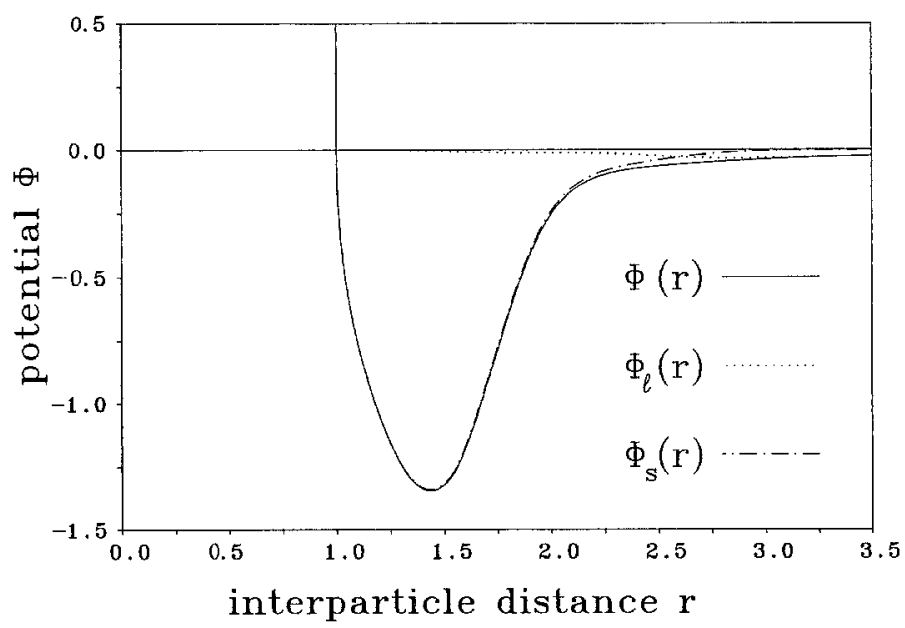

Fig. 2. Separation of a typical interparticle potential $\Phi(r)$ (solid line) into a short-ranged part $\Phi_{s}(r)$ (dotted line) and a long-ranged tail $\Phi_{l}(r)$ (dashed line) 


\section{Order parameters and lattice geometry}

Let us now consider a semi-infinite three-dimensional crystal in coexistence with its vapor and with a planar interface perpendicular to the $z$-axis. We parameterize the local density with a set of $z$-dependent order parameters, denoted by $\Gamma(z)=\left\{b_{0}(z), b_{1}(z), \ldots\right\}$. Hence, the local density may be written as $\rho(\mathbf{r}, \Gamma(z))$. We choose $b_{0}(z)$ to be the local mean density; the fields $b_{i}(z), i \geqq 1$, are local crystal order parameters such as Fourier coefficients (1.1) of the solid bulk density or functions thereof [27].

Our parameterization implies that for any spatially constant $\Gamma$, the local density $\rho(\mathbf{r}, \Gamma)$ possesses the symmetry of the bulk solid lattice, i.e. we have a fixed lattice on which the order parameters are superimposed. We assume that this lattice has inversion symmetry; the origin $\mathbf{r}=0$ is chosen at a lattice point.

For $z \rightarrow \pm \infty$, the order parameters must approach their solid or vapor bulk values. This requirement provides the boundary conditions

$$
\begin{aligned}
\Gamma(z \rightarrow \infty) & \equiv \Gamma_{S}=\left\{\rho_{S}, b_{1 S}, b_{2 S}, \ldots\right\}, \\
\Gamma(z \rightarrow-\infty) & \equiv \Gamma_{V}=\left\{\rho_{V}, 0,0, \ldots\right\} .
\end{aligned}
$$

Let $\left\{\mathbf{R}_{\mathbf{m}}\right\}$ denote the bulk lattice sites and $\left\{\mathbf{G}_{\mathbf{m}}\right\}$ the reciprocal lattice vectors. We set $\mathbf{m}=\left(\mathbf{m}^{\|}, n\right)$, where $n$ and $\mathbf{m}$ "label lattice vectors perpendicular and parallel to the plane interface, respectively. Such a representation exists for each orientation of the interface. The distance of two planar layers is denoted by $\bar{a}$. Furthermore, $V_{\mathbf{m}}$ is the elementary cell at $\mathbf{R}_{\mathrm{m}}$ and $V_{n}$ is a layer centered at $z_{n} \equiv n \bar{a}$, whose volume equals $\bar{a} A$ where $A$ is the surface area.

\section{Short-ranged forces: Gradient expansion}

We now assume that the order parameters are slowly varying over a lattice distance. Therefore, we may perform a gradient expansion in the short-range part $\Omega_{s}[\rho]$ of the functional $\Omega[\rho]$; the long-ranged part will be treated differently in the next section. We write $\Omega_{s}[\rho]$ as an integral of a free energy density $\omega_{s}([\rho], \mathbf{r})$ and split the integration into parallel layers

$\Omega_{s}[\rho]=\int \mathrm{d}^{3} r \omega_{s}([\rho], \mathbf{r})=\sum_{n} \int_{V_{n}} \mathrm{~d}^{3} r \omega_{s}([\rho], \mathbf{r})$.

The next step is to expand the integrand of (2.8) in each layer up to second order in the order parameters around the periodic reference density $\rho_{n}(\mathbf{r}) \equiv \rho\left(\mathbf{r}, \Gamma\left(z_{n}\right)\right)$. This yields

$$
\begin{aligned}
& \Omega_{s}[\rho] \cong \sum_{n} \int_{V_{n}} \mathrm{~d}^{3} r\left[\omega_{s}\left(\left[\rho_{n}\right], \mathbf{r}\right)+\int_{V} \mathrm{~d}^{3} r^{\prime} C\left(\mathbf{r} ; \mathbf{r}^{\prime} ;\left[\rho_{n}\right]\right)\right. \\
& \cdot\left\{\rho_{i}\left(\mathbf{r}^{\prime}, \Gamma\left(z_{n}\right)\right)\left[\dot{b_{i}}\left(z_{n}\right)\left(z^{\prime}-z_{n}\right)+\ddot{b}_{i}\left(z_{n}\right)\left(z^{\prime}-z_{n}\right)^{2} / 2\right]\right. \\
& \left.+\rho_{i j}\left(\mathbf{r}^{\prime}, \Gamma\left(z_{n}\right)\right) \dot{b_{i}}\left(z_{n}\right) \dot{b_{j}}\left(z_{n}\right)\left(z^{\prime}-z_{n}\right)^{2} / 2\right\} \\
& +\int^{3} \mathrm{~d}^{3} \mathrm{~d}^{3} \mathrm{~d}^{\prime \prime} C\left(\mathbf{r} ; \mathbf{r}^{\prime}, \mathbf{r}^{\prime \prime} ;\left[\rho_{n}\right]\right) \rho_{i}\left(\mathbf{r}^{\prime}, \Gamma\left(z_{n}\right)\right) \rho_{j}\left(\mathbf{r}^{\prime \prime}, \Gamma\left(z_{n}\right)\right) \\
& \left.\cdot \dot{b_{i}}\left(z_{n}\right) \dot{b_{j}}\left(z_{n}\right)\left(z^{\prime}-z_{n}\right)\left(z^{\prime \prime}-z_{n}\right) / 2\right] .
\end{aligned}
$$

Here, we defined

$C\left(\mathbf{r} ; \mathbf{r}_{1}, \ldots, \mathbf{r}_{k} ;[\rho]\right)=\delta^{k} \omega_{s}([\rho], \mathbf{r}) / \delta \rho\left(\mathbf{r}_{1}\right) \ldots \delta \rho\left(\mathbf{r}_{k}\right) ;(2.10)$

and $\rho_{i}=\partial \rho / \partial b_{i}, \rho_{i j}=\partial^{2} \rho / \partial b_{i} \partial b_{j:}$ Summation over repeated indices is understood and $\dot{b}_{i}=\partial b_{i} / \partial z$.

From lattice symmetry, we obtain

$$
\begin{aligned}
& C\left(\mathbf{r}+\mathbf{R}_{\mathbf{m}} ; \mathbf{r}_{1}+\mathbf{R}_{\mathbf{m}}, \ldots, \mathbf{r}_{k}+\mathbf{R}_{\mathbf{m}} ;\left[\rho_{n}\right]\right) \\
& \quad=C\left(\mathbf{r} ; \mathbf{r}_{1}, \ldots, \mathbf{r}_{k} ;\left[\rho_{n}\right]\right),
\end{aligned}
$$

and by inversion symmetry

$C\left(\mathbf{r},-\mathbf{r}_{1} ;\left[\rho_{n}\right]\right)=C\left(\mathbf{r} ; \mathbf{r}_{1} ;\left[\rho_{n}\right]\right)$.

Because of (2.11), the integration over $V_{n}$ in (2.9) reduces to that over $V_{0}$ such that the $z_{n}$-dependence is merely implicit via $b_{i}\left(z_{n}\right)$. Also, by (2.12), the term linear in $\dot{b}_{i}\left(z_{n}\right)$ vanishes.

As a next step, we take the continuum limit of (2.9),

$$
\sum_{n} \ldots \rightarrow(1 / \bar{a}) \int_{-\infty}^{\infty} \mathrm{d} z_{n} \ldots
$$

After partial integration of the term linear in $\ddot{b}_{i}$ and with

$(1 / \bar{a}) \int_{V_{0}} \mathrm{~d}^{3} r \ldots \rightarrow(A / V) \int \mathrm{d}^{3} r \ldots$,

we arrive at the following expression for the surface tension $\Sigma_{s}[\Gamma] \equiv \Omega_{s} / A$ due to short-range forces:

$\Sigma[\Gamma] \cong \int_{-\infty}^{\infty} \mathrm{d} z\left\{g_{i j}(\Gamma(z)) \dot{b}_{i}(z) \dot{b}_{j}(z)-v_{s}(\Gamma(z))\right\}$,

where

$\Omega_{s}[\rho(\mathbf{r}, \Gamma)] / V=-v_{s}(\Gamma)$

is the grand canonical free energy density for a system with spatially constant order parameters. The "metric tensor" $g_{i j}$ is given by

$$
\begin{gathered}
g_{i j}(\Gamma)=1 /(2 \beta V) \int \mathrm{d}^{3} r \mathrm{~d}^{3} r^{\prime} c_{s}\left(\mathbf{r}, \mathbf{r}^{\prime}, \Gamma\right) \\
\cdot \rho_{i}(\mathbf{r}, \Gamma) \rho_{j}(\mathbf{r}, \Gamma)\left(z-z^{\prime}\right)^{2}
\end{gathered}
$$

where $c_{s}$ denotes the DCF with spatially constant order parameters $\Gamma$.

\section{Long-ranged forces}

The length scale for the order parameter gradients is determined by the second moment of the DCF in (2.17). The weak long-ranged tail $\Phi_{l}(r)$ varies slowly on this scale, which is on the order of a few hard core diameters. Therefore, a gradient expansion in $F_{l}[\rho]$ is inadequate. Formally, this may also be seen by the fact that higher moments of $\Phi_{l}(r)$ do not exist.

In order to proceed, we take, for the moment, the order parameters to be the Fourier coefficients $b_{\mathbf{G}}(z)$ of the solid bulk density, 
$\rho(\mathbf{r}, \Gamma(z))=\sum_{\mathbf{G}} b_{\mathbf{G}}(z) \exp (\mathrm{i} \mathbf{G} \cdot \mathbf{r})$,

with the local mean density $b_{0}(z)$. We write $\mathbf{G}=\left(\mathbf{G}^{\|}, G^{\perp}\right)$, with components parallel and perpendicular to the interface. In $F_{i}[\rho]$, terms involving coefficients $b_{\mathbf{G}}$ with $\mathbf{G}^{\|} \neq 0$ are found to be negligible [28], so that the surface tension $\Sigma_{l}=F_{l} / A$ is approximated by

$\Sigma_{l} \cong \sqrt{(\pi / 2)} \int_{-\infty}^{\infty} \mathrm{d} k \tilde{w}(k)\left|\sum_{G^{\perp}} \tilde{b}_{\left(0, G^{\perp}\right)}\left(k+G^{\perp}\right)\right|^{2}$

with $\tilde{b}$ and $\tilde{w}$ denoting the Fourier transforms of $b_{\mathbf{G}}(z)$ and $w(z)$. The latter quantity is the laterally integrated potential,

$w(z)=\int_{A} \mathrm{~d}^{2} r_{\|} \Phi_{l}\left(\sqrt{r_{\|}^{2}+z^{2}}\right)$.

Since $w(z)$ and $b_{\mathbf{G}}(z)$ with $\mathbf{G}=\left(0, G^{\perp}\right)$ are both slowly varying over lattice distances, their Fourier transforms are concentrated in the first Brillouin zone $[-\pi / a, \pi / a]$. Therefore, the $G^{\perp}=0$ term dominates the sum on $G^{\perp}$ in (2.19), so that

$\Sigma_{l} \cong(1 / 2) \int_{-\infty}^{\infty} \mathrm{d} z \int_{-\infty}^{\infty} \mathrm{d} z^{\prime} w\left(\left|z-z^{\prime}\right|\right) b_{0}(z) b_{0}\left(z^{\prime}\right)$.

Within this approximation, the interaction via $\Phi_{l}(r)$ only involves the local mean density.

\section{Interfacial free energy}

After collection of terms, we obtain the following expression for the interfacial free energy (surface tension):

$$
\begin{aligned}
\Sigma[\Gamma]= & \int_{-\infty}^{\infty} \mathrm{d} z\left[g_{i j}(\Gamma(z)) \dot{b_{i}}(z) \dot{b}_{j}(z) / 2-v(\Gamma(z))\right] \\
& -\int_{-\infty}^{\infty} \mathrm{d} z \int_{-\infty}^{\infty} \mathrm{d} z^{\prime} w\left(\left|z-z^{\prime}\right|\right)\left(b_{0}(z)-b_{0}\left(z^{\prime}\right)\right)^{2} / 4 .
\end{aligned}
$$

Here, $-v(\Gamma)$ is the grand canonical free energy per unit volume of a system with spatially uniform order parameters $\Gamma$, including short-ranged as well as long-ranged forces.

The Eq. (2.22) resembles the mechanical action of a fictitious particle in $\Gamma$-space with metric $g_{i j}(\Gamma)$ and "time" $z$. The particle moves in a potential $v(\Gamma)$ with boundary conditions (2.7). This dynamical analogy in the context of interfacial phenomena is well known [29]. In the present case, its intuitive appeal is somewhat spoiled by the quadratic term in $b_{0}$-direction, which is non-local in the "time" $z$. However, a similar non-local contribution to the action arises in polaron models via the coupling of a particle to a boson field [30].

The equilibrium profile of the order parameters $\Gamma(z)$ is calculated by minimizing the surface tension $\Sigma(\Gamma)$. In the dynamical terminology, we have to solve the Euler equations of motion,

$\delta \Sigma / \delta b_{i}(z)=0$,

with the boundary conditions (2.7).

Let $\bar{\Gamma}(z)=\left(\bar{b}_{0}(z), \bar{b}_{1}(z), \ldots\right)$ be a solution of $(2.23)$, obeying the boundary conditions. The associated surface tension becomes

$$
\begin{aligned}
\Sigma[\bar{\Gamma}]= & \int_{-\infty}^{\infty} \mathrm{d} z\left\{\overline { b } _ { i } ( z ) \left[\partial v(\bar{\Gamma}(z)) / \partial b_{i}-(1 / 2){\stackrel{\circ}{b_{k}}}_{k}(z){\stackrel{\circ}{b_{j}}}_{j}(z)\right.\right. \\
& \left.\left.\cdot \partial g_{k j}(\bar{\Gamma}(z)) / \partial b_{i}\right] / 2-v(\bar{\Gamma}(z))\right\} .
\end{aligned}
$$

This form of $\Sigma[\bar{\Gamma}]$ is derived by using the virial theorem. It is remarkable, that the non-locality in $z$ only enters in (2.24) implicity via the profile $\Gamma(z)$.

For physical reasons we expect that the Euler equations (2.23) will have at least one solution. If we find more than one trajectory $\Gamma(z)$ solving (2.23) and obeying the boundary conditions (2.7), we have to select that one with minimal surface tension.

\section{Interfacial profiles from short-ranged potentials}

In this chapter we discuss qualitative properties of the solutions of (2.23), which yield a density profile of the solid|vapor interface wetted by an incipient liquid layer. Let us first restrict attention to purely short-ranged potentials. In this case, the Euler equations (2.23) read explicitly

$$
\begin{aligned}
E_{n}([\Gamma], z) \equiv & g_{n i}(\Gamma(z)) \ddot{b}_{i}(z)+\Gamma_{n i j}(\Gamma(z)) \dot{b}_{i}(z) \dot{b}_{j}(z) \\
& +\partial v / \partial b_{n}=0,
\end{aligned}
$$

where

$2 \Gamma_{n i j}=\partial g_{n i} / \partial b_{j}-\partial g_{i j} / \partial b_{n}+\partial g_{n j} / \partial b_{i}$

are the Christoffel symbols of the metric $g_{i j}$.

We consider first the simple case of only two order parameters $(N=2)$. Most of the results for $N=2$ can be taken over to the case of general $N$, which we examine in Sect. III.2.

\section{Two order parameters}

Square-gradient theories involving two order parameters with constant and diagonal mass tensor have been applied previously to studies of the Potts model [32], orderdisorder transitions in $\mathrm{Cu}_{3} \mathrm{Au}$ [33] and surface melting [12]. In this section, we summarize and occasionally extend those results which are relevant in the present context.

The form of the potential $v(\Gamma), \Gamma=\left(b_{0}, b_{1}\right)$ is assumed to be as follows: Near the triple point $T_{T}$, it exhibits a three-peak-structure with two maxima of equal height at $\Gamma_{V}=\left(\rho_{V}, 0\right)$ and $\Gamma_{S}=\left(\rho_{S}, b_{1 S}\right)$, as well as a lower maxi- 
mum at $\Gamma_{L}=\left(\rho_{L}, 0\right)$ rising with reduced temperature,

$t \equiv\left(T_{T}-T\right) / T_{T}$,

so that $v\left(\Gamma_{S}\right)=v\left(\Gamma_{V}\right)=v\left(\Gamma_{L}\right)$ for $t=0$. Here, $\rho_{L}$ is the liquid density at the triple point and we assume

$\rho_{V}<\rho_{L}<\rho_{S}$

In the terminology of dynamics, we look for trajectories which start on the top of the vapor peak and terminate on top of the solid peak.

As mentioned before, (3.1) with boundary conditions (2.7) may have several solutions. One of them is a "melting'-trajectory which passes near the liquid peak and approaches its top as $t \rightarrow 0$. In addition, other solutions may exist, describing 'blocked melting' or 'non-melting', see Fig. 3. Below it is shown that the number of solutions, $N_{\text {ext }}$, is odd.

In the vicinity of the peaks, the solutions of (3.1) resemble trajectories of a linearized dynamical system near hyperbolic fixed points. The correct asymptotic behavior of these trajectories can be deduced from (3.1) by approximating $v(\Gamma)$ with paraboloids and taking $g_{i j}(\Gamma)$ to be constant, see e.g. [34]. At the vapor and metastable liquid peak, the mass tensor is diagonal,

$g_{i j}(\Gamma) \cong g_{i}^{J} \delta_{i j}$

and $v(\Gamma)$ becomes

$v(\Gamma) \cong-\lambda_{y}^{2}\left(b_{0}-\rho_{J}\right)^{2} / 2-\mu_{J}^{2} b_{1}^{2} / 2-a \delta_{J L} t$,

for $J=V, L$. Notice that [12]

$\mu_{V} \gg \mu_{L}>0$.

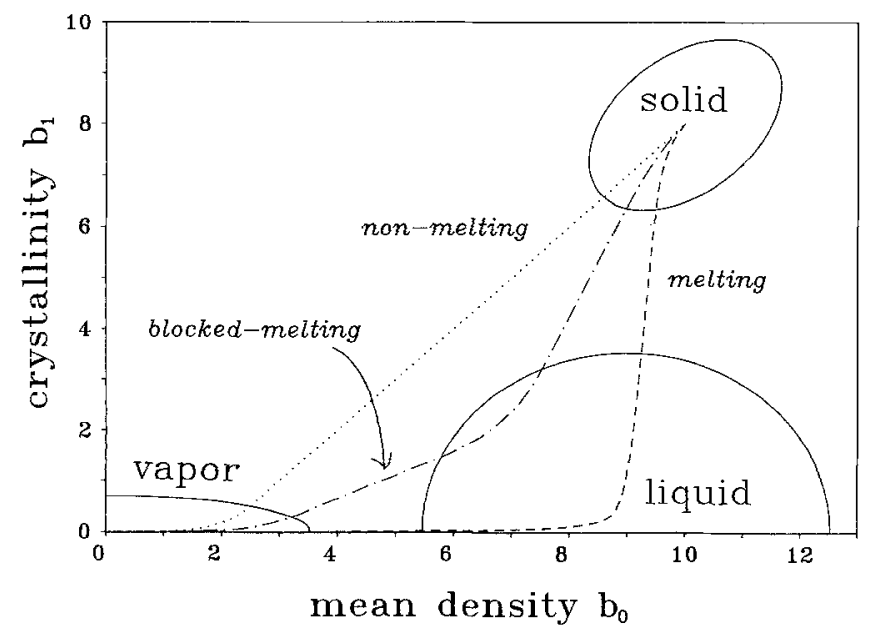

Fig. 3. Illustration of three types of solutions in order parameter space: (i) melting (dashed line), (ii) blocked-melting (dot-dashed line), (iii) non-melting (dotted line). We have chosen one crystallinity order parameter $b_{1}$. The units are arbitrary. The mass tensor is taken as diagonal and constant $g_{i j}=\delta_{i j}$. The potential $v$ is the maximum of three inverted paraboloids and the plane $p(\Gamma)$, defined by $p(\Gamma) \equiv-4$. The ellipses are the intersection lines of $p(\Gamma)$ with the paraboloids. The reduced temperature is chosen as $a \cdot t=0.01$

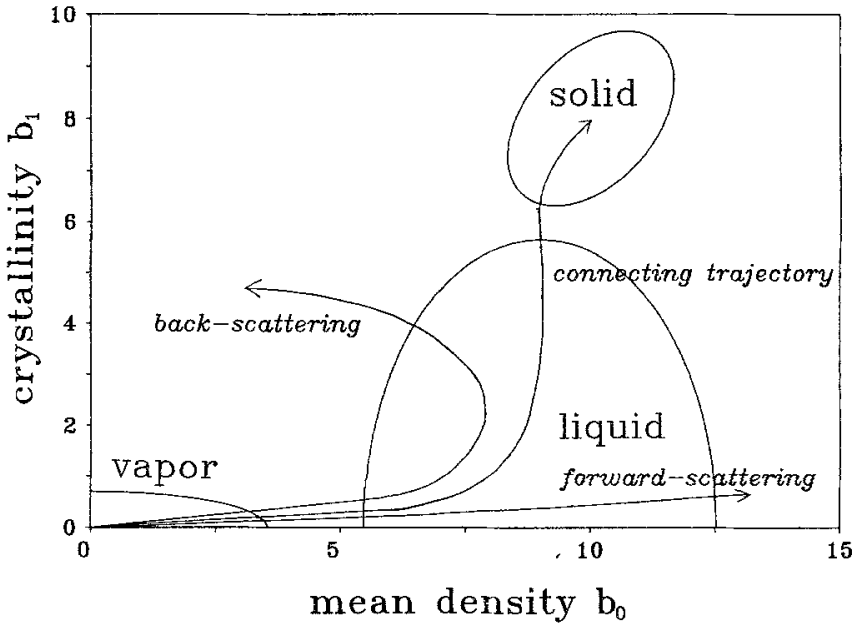

Fig. 4. Qualitative picture of forward and back scattering at the liquid peak for different initial conditions. Each trajectory starts at the top of the vapor peak

In the vicinity of the solid peak, $g_{i j}$ and the quadratic approximant of $v(\Gamma)$ will be non-diagonal in general.

The linearized equations of motion can now be easily solved around $\Gamma=\Gamma_{V}, \Gamma_{S}, \Gamma_{L}$. One finds that the order parameters approach their bulk vapor or solid value exponentially with a non-universal decay length. For instance,

$b_{0}(z)-\rho_{V} \sim \exp \left(-\lambda_{V} / \sqrt{g_{0}^{V}}|z|\right)$ as $z \rightarrow-\infty$.

A more detailed discussion of the decay lengths will be given in [35].

As a second application, we examine trajectories which start from the vapor peak and are scattered at the liquid peak, as illustrated in Fig. 4. Consider the initial conditions

$\Gamma\left(z_{0}\right)=\left(b_{0}^{0}, b_{1}^{0}\right), \quad \dot{\Gamma}\left(z_{0}\right)=\left(\dot{b}_{0}^{0}, \dot{b}_{1}^{0}\right)$.

Then, for $z \gg z_{0}$,

$b_{i}(z) \cong \rho_{L} \delta_{i 0}+A_{i} \exp \left(\omega_{i}\left(z-z_{0}\right)\right) \quad(i=0,1)$

with $\omega_{0}=\lambda_{L} / \sqrt{g_{0}^{L}}, \omega_{1}=\mu_{L} / \sqrt{g_{1}^{L}}$ and $A_{i}=b_{i}^{0}+\dot{b}_{i}^{0} / \omega_{i} . A_{1}$ is positive, but the sign of $A_{0}$ may change. Since the trajectories start at the vapor peak, we have

$\dot{b}_{0}^{0} \cong\left(\lambda_{V} / \sqrt{g_{0}^{V}}\right) b_{0}^{0}, \quad \dot{b}_{1}^{0} \cong\left(\mu_{V} / \sqrt{g_{1}^{V}}\right) b_{1}^{0}$.

Then, conservation of 'energy' in the fictitious dynamics implies for small $b_{1}^{0}$ and $t$, that

$A_{0} \cong 2 a t-D\left(b_{1}^{0}\right)^{2}$,

with $D>0$, because of (3.7). Thus, $A_{0}$ is positive for very small $b_{1}^{0}$ and becomes negative for $b_{1}^{0} \cong \sqrt{2 a t / D}$. As $b_{1}^{0}$ increases the trajectories change from forward scattering to back scattering, see Fig. 4. Consequently, there exists at least one trajectory which approaches the solid peak 
for $z \rightarrow \infty$. This is a melting solution, since its initial value $b_{1}^{0}(t)$ vanishes as $t \rightarrow 0$.

Physically, $b_{1}^{0}(t)$ provides a measure for the residual crystallinity at the quasi-liquid-vapor-interface. After eliminating $z$ in (3.9), we find that $b_{1}^{0}(t) \sim t^{\nu}$ with

$y=\max \left(1 / 2, \omega_{1} / \omega_{0}\right)$

Therefore, the length $l$ of the quasi-liquid layer (which corresponds to the resting time of the fictitious particle on the liquid peak) diverges logarithmically

$l(t) \sim-\ln t, \quad t \rightarrow 0$.

The surface tension behaves as $t \ln t$ and the surface specific heat $c_{s}(t)$ diverges as

$c_{s}(t) \sim t^{-1}, \quad t \rightarrow 0$.

The asymptotic laws (3.13) and (3.14) are well-known [36], but (3.12) is a new result.

Let us now demonstrate that the number $N_{\text {ext }}$ must be odd. Consider the set of solutions of (3.1) which start at the vapor peak for $z \rightarrow-\infty$. These trajectories can be continuously parameterized by $0 \leqq \alpha \leqq \infty$, such that the velocities of the solutions satisfy

$$
\begin{array}{ll}
\lim _{z \rightarrow-\infty} \dot{b}_{1}(z) / \dot{b}_{0}(z)=0 & \text { for } \alpha=0 \\
\lim _{z \rightarrow-\infty} \dot{b}_{0}(z) / \dot{b}_{1}(z)=0 & \text { for } \alpha=\infty .
\end{array}
$$

Furthermore, consider a fixed equipotential line $L$ in $\left(b_{0}, b_{1}\right)$-space near the solid peak. For certain values of $\alpha \in \mathscr{A} \subseteq[0, \infty]$ the solutions intersect the line $L$. We consider the angle $\phi(\alpha)$ between the velocity of the solution at the intersection point and the velocity which is actually required to reach the top of the solid peak from the intersection point. The function $\phi(\alpha)$ is piecewise continuous and does not change sign at a discontinuity. Moreover $\phi(\alpha)$ is continuous in $\alpha_{0}$, where $\phi\left(\alpha_{0}\right)=0$, which is the condition for a connecting trajectory. Furthermore, if $\alpha_{-} \equiv \inf \mathscr{A}$ and $\alpha_{+} \equiv \sup \mathscr{A}$, we know from the discussion above, that sign $\phi\left(\alpha_{-}\right)=-\operatorname{sign} \phi\left(\alpha_{+}\right)$. This shows, that $\phi(\alpha)$ has, in general, an odd number of zeroes, i.e. $N_{\mathrm{ext}}$ is an odd number.

Thus, we have shown that the number of connecting solutions is odd, one of which is a melting trajectory.

For $N_{\text {ext }}=3$, a non-melting and a blocked-melting solution occur, as shown in Fig. 3. If the potential has some substructure besides the three main peaks, then $N_{\text {ext }}>3$ may be possible. For $N_{\text {ext }}>1$, the physically realized trajectory is that one with minimal "action' $\Sigma$ in the form (2.24). For $w=0$, however, energy conservation leads to the simpler expression

$\Sigma=-2 \int_{-\infty}^{\infty} \mathrm{d} z v(\bar{\Gamma}(z))$

where $\bar{\Gamma}(z)$ is a solution of (3.1). The Eq. (3.15) implies that a crossing of a deep valley between the peaks is costly in surface energy and therefore is unfavorable. We found numerically that the blocked melting solution is normally unstable for physically reasonable potentials $v(\Gamma)$. However, we have no compelling argument to exclude in general substructures in $v(\Gamma)$, which would render the blocked melting trajectory into a stable one.

\section{Arbitrary number of order parameters}

In order to obtain a surface tension depending on the orientation of the crystal surface plane at least three order parameters are required. Qualitative features, such as the growth law of the quasi-liquid film, the residual crystallinity and the asymptotic behavior of the order parameters are the same as discussed in Sect. III.1. In particular, for the residual crystallinity $b_{i}(t) \sim t^{\nu_{i}}$ we have

$v_{i}=\operatorname{Max}\left(\omega_{i} / \omega_{0}, \omega_{i} /\left(2 \omega_{1}\right), \ldots, \omega_{i} /\left(2 \omega_{N-1}\right)\right)$,

where $\omega_{i}=\mu_{i}^{L} / \sqrt{g_{i}^{L}}$, with $\left(\mu_{i}^{L}\right)^{2}$ denoting the curvature of the potential $v(\Gamma)$ at the liquid peak in $b_{i}$ direction and $g_{i}^{L}$ is the $i$-th element of the mass tensor, $i=1, \ldots, N-1$. We assumed that the potential and the mass tensor are diagonal in the liquid, which can be achieved for a suitable choice of crystallinity OP. This is the case, for instance, if the $b_{i}(z)$ are the Fourier coefficients of the bulk solid density according to (2.18).

We note that, the proof for $N_{\text {ext }}=$ odd no longer applies, since the two-dimensionality of the order parameter space was essential for the argument.

\section{Long-ranged potentials}

With long-ranged potentials included, the equations of motion (2.23) read

$$
\begin{aligned}
& E_{n}([\Gamma], z)-\delta_{n 0} \int_{-\infty}^{\infty} \mathrm{d} z^{\prime} w\left(\left|z-z^{\prime}\right|\right) \\
& \cdot\left(b_{0}\left(z^{\prime}\right)-b_{0}(z)\right)=0, \quad n=0, \ldots, N-1
\end{aligned}
$$

where $E_{n}([\Gamma], z)$ is defined in (3.1).

\section{An exactly solvable model}

Let us first consider a simplified model with only one crystallinity order parameter. The mass tensor is assumed to be diagonal and constant,

$g_{i j}(\Gamma)=g_{i} \delta_{i j}, \quad i, j=0,1$

and the potential $v(\Gamma)$ is approximated by three truncated paraboloids

$$
\begin{aligned}
& v(\Gamma)=-\frac{1}{2} \operatorname{Min}\left[\lambda^{2}\left(b_{0}-\rho_{V}\right)^{2}+\mu_{V}^{2} b_{1}^{2} ;\right. \\
& 2 a t+\lambda^{2}\left(b_{0}-\rho_{L}\right)^{2}+\mu_{L}^{2} b_{1}^{2} ; \\
& \left.\lambda^{2}\left(b_{0}-\rho_{S}\right)^{2}+\mu_{S}^{2}\left(b_{1}-b_{S}\right)^{2}\right]
\end{aligned}
$$


with $\rho_{S}>\rho_{L}>\rho_{V}>0$ and $\mu_{V}>\mu_{S}>\mu_{L}>0$. Note that the curvatures in $b_{0}$-direction are assumed to be equal in each phase.

The long-ranged potential $w(z)$ will not be specified in order to discuss the effects of different choices of $w(z)$ on the residual crystallinity and on the width of the quasi-liquid layer in the melting trajectory.

We also note that the solutions of (4.1) can be extended straightforwardly within this simplified model to include more than one crystal order parameter.

A. Sublimation trajectory. The interfacial profile along the sublimation curve well below the triple point is obtained from (4.1) in the formal limit $t \rightarrow \infty$. We are looking for a solution which connects the top of the solid paraboloid with the top of the vapor paraboloid. The interface is located on this sublimation trajectory, $\hat{\Gamma}(z)$, at the point, $z_{0}$, where $\hat{\Gamma}(z)=\left(\hat{b}_{0}\left(z_{0}\right), \hat{b}_{1}\left(z_{0}\right)\right)$ traverses the intersection line of the paraboloids. Since the Eqs. (4.1) are translational invariant in $z$, the location of the interface on the $z$-axis can be chosen freely and we fix $z_{0}=0$. Then, the Eqs. (4.1) are linear for $z<0$ and for $z>0$. At $z=0$, the 'potential' $v(\Gamma)$ gives rise to a step-like external 'force'. The explicit expression for $\hat{b}_{0}(z)$ is given in terms of a Fourier integral. After matching the solutions of the piecewise linear differential equations for $\hat{b}_{1}(z)$ we arrive at

$$
\begin{aligned}
\hat{b}_{0}(z)= & \rho_{S}+\rho_{V} / 2+\lambda^{2}\left(\rho_{S}-\rho_{V}\right) \pi^{-1} \\
& \cdot \int_{0}^{\infty} \mathrm{d} k \sin (k z)\left[k\left(g_{0} k^{2}+\lambda^{2}+W(k)\right)\right]^{-1},
\end{aligned}
$$

and

$$
\begin{aligned}
& \hat{b}_{1}(z)= \\
& \begin{cases}\mu_{S} b_{S} \exp \left(\mu_{V} z / \sqrt{g_{1}}\right) /\left(\mu_{S}+\mu_{V}\right), & \text { for } z \leqq 0 \\
b_{S}-\mu_{V} b_{S} \exp \left(-\mu_{S} z / \sqrt{g_{1}}\right) /\left(\mu_{S}+\mu_{V}\right), & \text { for } z>0\end{cases}
\end{aligned}
$$

where

$W(k)=\sqrt{2 \pi}(\tilde{w}(k)-\tilde{w}(0))$

with $\tilde{w}(k)$ denoting the Fourier transform of $w(z)$.

B. Melting trajectory. Now we turn to temperatures slightly below the triple point where $t$ in (4.3) is small. As in $(A)$, the nonlinearity of $v$ in order parameter space can be transformed into an external force by fixing $z=0$ on the intersection of the vapor and liquid paraboloid and $z=l$ on the solid-liquid intersection line. Hence, $l>0$ denotes a 'resting time' on the liquid paraboloid. For a given $l$, the solution $\bar{b}_{0}(z)$ of the melting trajectory $\bar{\Gamma}(z)=\left(\vec{b}_{0}(z), \bar{b}_{1}(z)\right)$ reads

$\bar{b}_{0}(z)=\rho_{L} \widehat{b}_{0}(z) / \rho_{S}+\left(\rho_{S}-\rho_{L}\right) \hat{b}_{0}(z-l) / \rho_{S}$

with $\hat{b}_{0}(z)$ from (4.4).

The piecewise linear Euler equations involving $\hat{b}_{1}(z)$ are easily solved. After matching the solutions, we arrive at a relation $l=l(t)$ for a connecting trajectory which has to be inserted in (4.7). The calculation is straightforward but lengthy and we only present the results:

$$
\begin{aligned}
a t= & F(l)+Z G(l) / 2+Y G^{2}(l) \\
& -\sqrt{Y(Z-Y) G^{2}(l)+Z Y G^{3}(l)+Y^{2} G^{4}(l)}
\end{aligned}
$$

where $Z=b_{S}^{2} \mu_{S}^{2}\left(\mu_{S}^{2}+\mu_{L}^{2}\right) X, \quad Y=\mu_{S}^{4} b_{S}^{2} X, \quad X^{-1}=\mu_{S}^{2}-\mu_{L}^{2}$ and

$$
\begin{aligned}
F(l) & =\lambda^{2}\left(\rho_{S}-\rho_{L}\right)\left(\rho_{L}-\rho_{V}\right)\left(\rho_{S}-\hat{b}_{0}(l)\right) /\left(\rho_{S}-\rho_{V}\right), \\
G^{-1}(l) & =\left(\mu_{S}^{2}-\mu_{L}^{2}\right) g^{2}(l) /\left(\mu_{V}^{2}-\mu_{L}^{2}\right)-1,
\end{aligned}
$$

with $g(l)=\cosh (\omega l)+\mu_{V} \sinh (\omega l) / \mu_{L}$ and $\omega=\mu_{L} / \sqrt{g_{1}}$.

The solution $\bar{b}_{1}(z)$ is found to be

$\bar{b}_{1}(z)= \begin{cases}D \exp \left(\mu_{Y} z / \sqrt{g_{1}}\right), & z<0, \\ D g(z), & 0 \leqq z \leqq l, \\ b_{s}-\left(b_{s}-H\right) \exp \left(-\mu_{S}(z-l) / \sqrt{g_{1}}\right), & z>l,\end{cases}$

with $\quad D^{2}=2 X(a t-F(l)) \quad$ and $\quad H=Y /\left(\mu_{S}^{2} b_{S}\right)$ $-\sqrt{X\left(Y-\mu_{S}^{2} b_{S}^{2}+2(a t-F(l))\right)}$.

In (4.9), the width of the quasi-liquid layer $l$ depends on $t$ via Eq. (4.8). In general, Eq. (4.8) may have several solutions in $l$ for a given $t$. These solutions are either 'melting' or 'blocked melting' trajectories mentioned in Sect. III.

C. Discussion. From Eqs. (4.5) and (4.9) it is seen that crystal order parameters $\hat{b}_{1}(z)$ and $\bar{b}_{1}(z)$ approach their bulk solid and gas values exponentially, as in the shortrange case. On the other hand, the asymptotic behavior of $\bar{b}_{0}(z), \bar{b}_{0}(z)$ and $l(t)$ is governed by the singularities in $\tilde{w}(k)$ which arise from algebraic tails in $\Phi_{l}(r)$. For an attractive $\Phi_{l}(r) \cong-\alpha r^{-n}, \alpha>0, n>3$, we find that Eq. (4.8) has only one solution, which is a melting trajectory. The width of the quasi-liquid layer increases according to

$l(t) \cong L_{0} t^{-1 /(n-3)}$,

and the surface specific heat diverges as

$c_{s}(t) \sim t^{-(n-2) /(n-3)}$.

These growth laws are the same as those found for sharpkink models [36]. The mean density approaches its bulk values as $z^{-(n-3)}$.

The residual crystallinity at the interface of the quasiliquid layer and the vapor phase may be defined as $b_{1}^{0}(t)=\bar{b}_{1}(z=0, t)$; we obtain

$b_{1}^{0}(t) \sim \exp \left(-\omega L_{0} t^{-1 /(n-3)}\right)$.

This stretched exponential form for $b_{1}^{0}(t)$ is a novel analytic result; it agrees with numerical findings of Trayanov and Tosatti [10]. The prefactor $L_{0}$ in (4.10) and (4.12) is related to the Hamaker constant by $L_{0}^{3}=2 W / a$. For $n=6$ (Lennard-Jones potential) we have

$L_{0}^{3}=\left(\rho_{S}-\rho_{L}\right)\left(\rho_{L}-\rho_{V}\right) \rho_{S} \pi \alpha /\left[6 a\left(\rho_{S}-\rho_{V}\right)\right]$. 


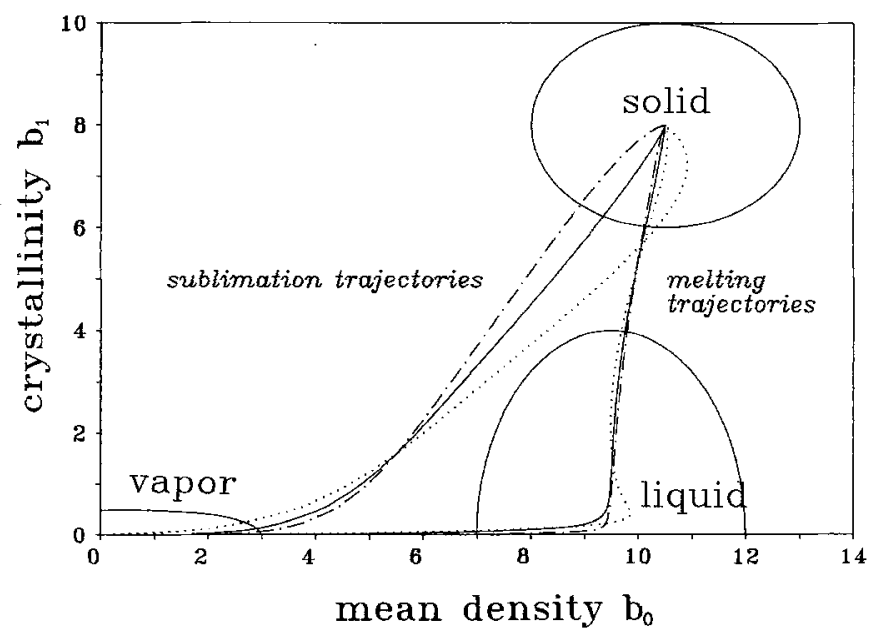

Fig. 5. Melting $(t=0.0033)$ and sublimation $(t \rightarrow \infty)$ trajectories in order parameter space in arbitrary units. We include long-ranged forces with $w(z)=\gamma /\left(\sigma^{2}+z^{2}\right)^{2}$, corresponding to $\Phi_{l}(r) \sim r^{-6}$. The solid line is for a short-ranged potential $(\gamma=0)$ whereas the dot-dashed and the dotted line correspond to a long-range attractive $(\gamma=-1)$ and repulsive $(\gamma=1)$ potential. For $\gamma=1$, the blocked melting trajectory is shown. The ellipses are given by $v(\Gamma)=-2$. We choose $\mathrm{g}_{0}=1, \mathrm{~g}_{1}=0.4854, a=1, \sigma=1, \lambda=0.8, \mu_{V}=4, \mu_{L}=0.5, \mu_{S}=1, \rho_{V}$ $=0.5, \rho_{L}=0.95, \rho_{S}=10.5, b_{S}=8$

The sublimation and melting trajectories are displayed in Fig. 5. The temperature dependence of the width $l(t)$ is shown in Fig. 6. For weak long-ranged potentials, the function $l(t)$ exhibits a cross-over from the logarithmic law of the short-range case to an algebraic growth at some finite (reduced) temperature $t_{0}$, see inset of Fig. 6. Such a behavior of $l(t)$ has been observed in recent measurements on $\mathrm{Ne}[6]$ and $\mathrm{Pb}$ [4]. This change in the character of $l(t)$ also induces a cross-over in the residual crystallinity.

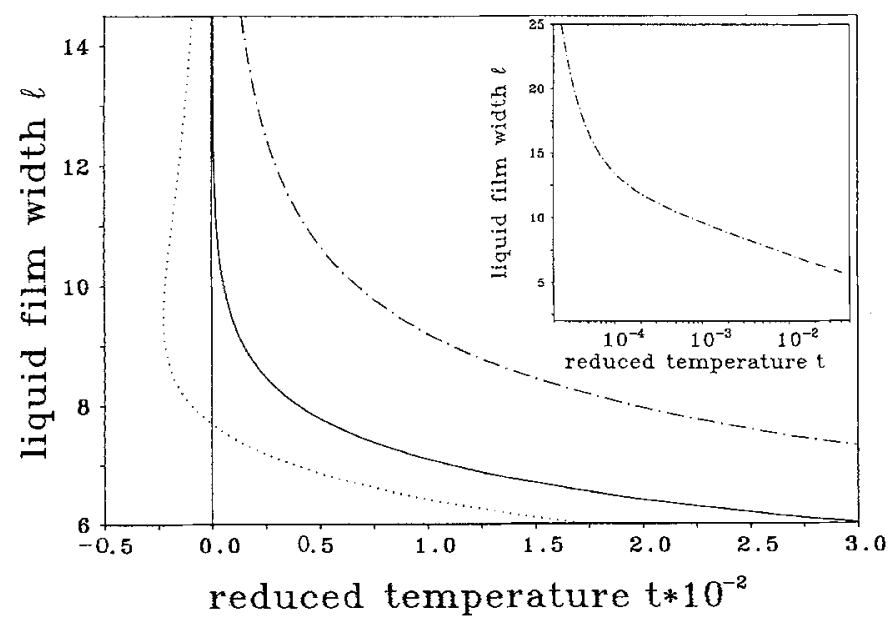

Fig. 6. Width of the liquid film versus reduced temperature $t$ in arbitrary units for different kinds of interparticle potentials. The line type and the parameters are as in Fig. 5. The inset shows the crossover from a logarithmic to an algebraic growth law for a weak attractive long-range potential $(\gamma=0.05)$
In order to exhibit the dependence of the trajectories on the nature of $\Phi_{l}(r)$ we also examine repulsive and slowly varying oscillatory tails, although their physical relevance is perhaps remote.

A repulsive potential $\Phi_{l}(r) \cong \alpha r^{-n}, \alpha>0, n>3$, yields a single, blocked melting trajectory. The density profile $b_{0}(z)$ varies non-monotonously with $z$ and approaches its bulk values as $z^{-(n-3)}$, see Fig. 5 . The width $l(t)$ (see Fig. 6) and the surface specific heat $c_{s}(t)$ remain finite for $t \rightarrow 0$. The behavior of $l(t)$ in Fig. 6 for $t<0$ corresponds to a non-equilibrium situation with overheated solid and vapor.

In the case of an oscillatory tail $\Phi_{l}(r) \cong \alpha \cos \left(k_{0} r\right) / r^{n}$, $n \geqq 1$, with $k_{0}^{-1}$ much larger than lattice distance, the Eq. (4.8) can have several solutions, see Fig. 7. At some temperature $t_{0}$ there is a bifurcation and a layering transition to the upper branch with minimal surface tension. As $t$ decreases, additional solutions appear. However, there are no further layering transitions, since these solutions have higher surface tension than the branch ending at a finite width $l_{\max }$. Hence, we have again blocked surface melting. Figure 8 shows some examples of connecting trajectories with oscillatory $b_{0}(z)$, which approaches its bulk values as $\sim \cos \left(k_{0} z+\phi\right) / z^{n-1}, \phi$ denoting a phase.

\section{General case}

Let us first restrict attention to the case of two order parameters but general functions $v(\Gamma)$ and $g_{i j}(\Gamma)$. We assume that the potential $v(\Gamma)$ is invariant under inversion $b_{1} \rightarrow-b_{1}$ with $b_{0}$ kept fixed. Furthermore, let $g_{i j}(\Gamma)$ be diagonal for $b_{1}=0$. Then, in the vicinity of the vapor and liquid peak, the Euler equations decouple in leading order of the order parameters. Consequently, $b_{1}(z)$ ap-

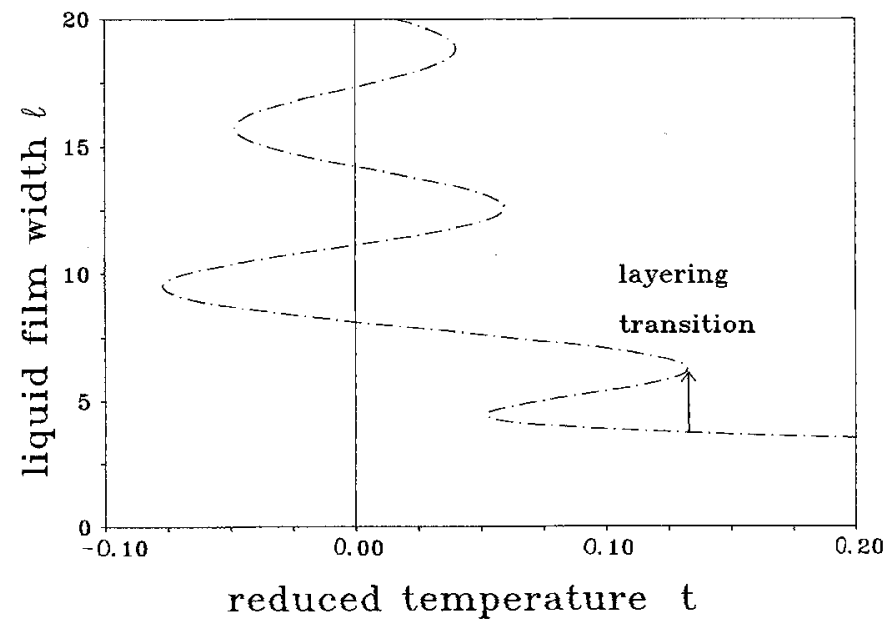

Fig. 7. Width of the liquid film versus reduced temperature $t$ in arbitrary units for an oscillating long-range potential $w(z)=$ $-\gamma \sin \left(k_{F} z\right) / z$, corresponding to $\Phi_{l} \sim \cos \left(k_{F} r\right) / r^{2}$. As parameters, we choose $g_{0}=0.48, g_{1}=1, k_{F}=1, \gamma=1$. The other parameters are as in Fig. 5. The layering transition is also indicated 
proaches the vapor/liquid peak exponentially with decay constant $\mu / \sqrt{g_{11}}$ with $\mu^{2}$ denoting the curvature of $v(\Gamma)$ in $b_{1}$-direction and $g_{11}$ the mass at the vapor/liquid peak. The asymptotic behavior of $\bar{b}_{0}(z)$ is again governed by the singularities of $\tilde{w}(k)$. Therefore, the results (4.10), (4.11) and the asymptotics of $\bar{\Gamma}(z)$ for $z \rightarrow-\infty$ remain unchanged for general $v\left(b_{0}, b_{1}\right), g_{i j}\left(b_{0}, b_{1}\right)$. However, since in general $g_{i j}$ and $v$ will be nondiagonal at the solid peak, the Euler equations for $\bar{b}_{0}(z)$ and $\bar{b}_{1}(z)$ are coupled and $\bar{b}_{1}(z)$ approaches the solid bulk value with the same algebraic law as $\bar{b}_{0}(z)$ does.

Now, we consider a set of crystal order parameters $\left\{b_{i}\right\}, i=1, \ldots, N-1$ with $g_{i j}(\Gamma)$ and the potential assumed to be diagonal for $b_{i}=0$. As already mentioned in Sect. III.2, this is the case for the Fourier coefficients of the bulk solid density, see (2.18). Then, by the same argument as above, (4.10) remains unaffected and (4.11) is generalized to

$b_{i}^{0}(t) \sim \exp \left(-\omega_{i} L_{0} t^{-1 /(n-3)}\right), \quad i=1, \ldots, N-1$

with $\omega_{i}=\mu_{i}^{L} / \sqrt{g_{i}^{L}}$ where $\left(\mu_{i}^{L}\right)^{2}$ is the curvature of $v$ in $b_{i}$-direction and $g_{i}^{L}=g_{i i}\left(\rho_{L}, 0,0, \ldots\right)$ is the 'mass' at the liquid peak.

We expect the number of solutions to be odd if $N=2$, even for long-ranged couplings. This conjecture is supported by the following arguments:

$i$. The melting trajectory of the short-ranged system can split up in an odd number of solutions as the long ranged interaction is turned on (compare the effect of an oscillating long-range tail).

ii. The long-ranged interaction will not alter a non-melting or blocked melting trajectory of the short-ranged system drastically, since it only smoothes it out.

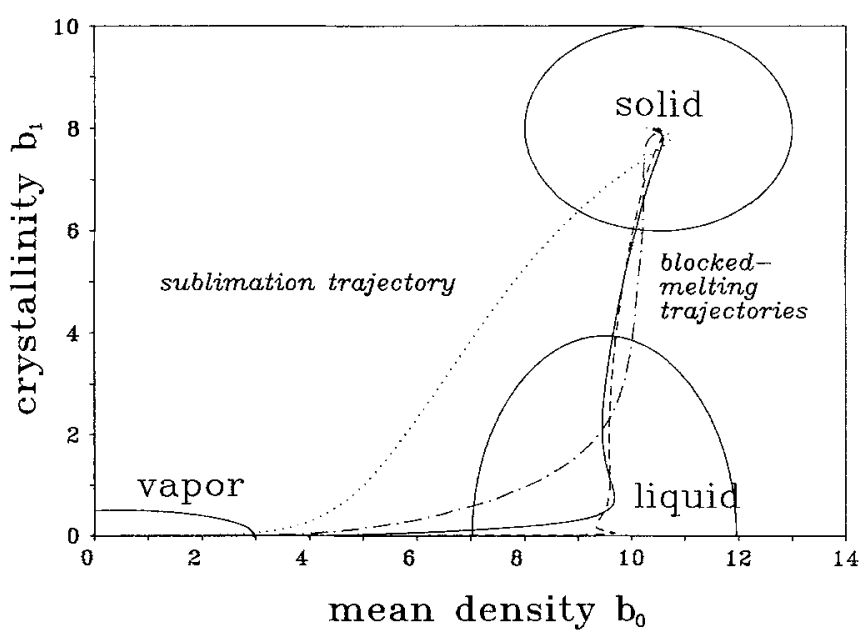

Fig. 8. Sublimation ( $t \rightarrow \infty$, dotted line) and blocked melting $(t=0.057)$ trajectories in order parameter space in arbitrary units for an oscillating tail. For $t=0.057$, there are five solutions which are blocked melting trajectories. Three of them are shown corresponding to $l=4.25$ (dot-dashed line), $l=7.54$ (solid line) and $l=12.9$ (dashed line). The solid line has minimal surface tension. The parameters are as in Fig. 7
What happens if $\rho_{L}>\rho_{S}$ ? Then, long-ranged attractive forces prevent melting since the sign of the Hamaker constant (4.13) changes. Thus only a blocked melting solution exists. Apparently, this was observed for Ge [37] whose liquid is indeed denser than the solid.

\section{Conclusions}

In this paper we described the first and more technical part of our work on surface melting i.e. the wetting of the solid/vapor interface by an incipient liquid layer. To provide a theoretical frame for this study, we constructed a variational expression for the interfacial free energy of an inhomogeneous system with a multicomponent order parameter. The effects of short-ranged as well as long-ranged interatomic forces are examined. The various length scales occuring in the structure of a wetted solid/vapor interface are incorporated consistently.

The Euler equations for the interfacial profile allow for melting, blocked melting and non-melting solutions. The asymptotic features of the melting solution in the vicinity of the triple point can be obtained in analytic form from a simple approximation for the local bulk free energy. In particular, we determined the decay of the residual crystalline order in the liquid layer; for weak long-ranged forces we find a crossover from logarithmic to power-law growth in $T_{T}-T$ of the interfacial width, in agreement with recent experiments $[4,6]$.

Surface melting also shows the well-known sensitivity of wetting phenomena upon the detailed form of the interparticle forces: repulsive long-ranged tails induce blocked melting; oscillatory tails lead to a layerwise blocked melting.

Our expression for the interface free energy is explicitly specified in microscopic terms and is practically calculable by utilizing current density functional techniques [25-27]. The results obtained within a weighted density approximation [31] will be given elsewhere.

We thank D. Kroll and S. Dietrich for helpful discussions. This work was supported by the Bundesministerium für Forschung und Technologie (BMFT) under contract 03WA1LMU5.

\section{References}

1. Frenken, J.W.M., Veen, J.F. van der: Phys. Rev. Lett. 54, 134 (1985)

2. Frenken, J.W.M., Maree, P.M.J., Veen, J.F. van der: Phys. Rev. B34, 7506 (1986)

3. Pluis, B., Denier van der Gon, A.W., Frenken, J.W.M., Veen, J.F. van der: Phys. Rev. Lett. 59, 2678 (1987)

4. Pluis, B., Taylor, T.N., Frenkel, D., Veen, J.F. van der: Phys. Rev. B 40, 1353 (1989)

5. Prince, K.C., Breuer, U., Bonzel, H.P.: Phys. Rev. Lett. 60, 1146 (1988)

6. Zhu, D.M., Dash, J.G.: Phys. Rev. Lett. 57, 2959 (1986); 60, $432(1988)$

7. Stranski, I.N.: Z. Phys. 119, 22 (1942)

8. For recent reviews on experiments of surface melting see e.g.: Veen, J.F. van der, Pluis, B., Denier van der Gon, A.W.: In: 
Chemistry and physics of solid surfaces. Vanselow, R., Howe, R.F. (eds.), p. 1701. Berlin, Heidelberg, New York: Springer 1988; Dash, J.G.: Contemp. Phys. 30, 89 (1989)

9. Pietronero, L., Tosatti, E.: Solid State Commun. 32, 255 (1979)

10. Trayanov, A., Tosatti, E.: Phys. Rev. Lett. 59, 2207 (1987); Phys. Rev. B38, 6961 (1988)

11. Lipowsky, R., Speth, W.: Phys. Rev. B28, 3983 (1983)

12. Levi, A.C., Tosatti, E.: Surf. Sci. 189-190, 641 (1987)

13. Lipowsky, R., Breuer, U., Prince, K.C., Bonzel, H.P.: Phys. Rev. Lett. 62, 913 (1989)

14. Yamamoto, T., Izuyama, T.: J. Phys. Soc. Jpn. 57, 3742 (1988)

15. Broughton, J.Q., Woodcock, L.V.: J. Phys. C11, 2743 (1978);

Broughton, J.Q., Gilmer, G.H.: J. Chem. Phys. 79, 5095 (1983); J. Chem. Phys. 79, 5105 (1983); J. Chem. Phys. 79, 5119 (1983)

16. Schommers, W.: Phys. Rev. B32, 6845 (1985)

17. Rosato, V., Ciccotti, G., Pontikis, V.: Phys. Rev. B33, 1860 (1986)

18. Valkealahti, S., Nieminen, R.M.: Phys. Scr. 36, 646 (1987)

19. Stoltze, P., Nørskov, J.K., Landman, U.: Phys. Rev. Lett. 61, $440(1988)$

20. Chen, E.T., Barnett, R.N., Landman, U.; Phys. Rev. B40, 924 (1989)

21. See e.g.: Baus, M.: J. Stat. Phys. 48, 1129 (1987); Haymet, A.D.J.: Prog. Solid State Chem. 17, 1 (1986)

22. Löwen, H., Beier, T., Wagner, H.: Europhys. Lett. 9, 791 (1989)

23. Evans, R.: Adv. Phys. 28, 143 (1979)

24. Weeks, J.D., Chandler, D., Anderson, H.C.: J. Chem. Phys. 54, 5237 (1971)
25. Tarazona, P.: Phys. Rev. A 31, 2672 (1985);

Curtin, W.A., Ashcroft, N.W.: Phys. Rev. A 32, 2909 (1985)

26. Curtin, W.A., Ashcroft, N.W.: Phys. Rev. Lett. 56, 2775 (1986)

27. Haymet, A.D.J., Oxtoby, D.W.: J. Chem. Phys. 74, 2559 (1981)

28. Gompper, G., Kroll, D.M.: Phys. Rev. B40, 7221 (1989)

29. Kampen, N.G. van: Phys. Rev. 135, A 362 (1964); see also: Widom, B.: In: Statistical mechanics and statistical methods in theory and applications. Landman, U. (ed.). New York: Plenum Press 1977

30. See e.g.: Bray, A.J., Moore, M.A.: Phys. Rev. Lett. 49, 1545 (1982)

31. Denton, A.R., Ashcroft, N.W.: Phys. Rev. A39, 4701 (1989)

32. Hauge, E.H.: Phys. Rev. B33, 3322 (1986)

33. Gompper, G., Kroll, D.M.: Phys. Rev. B36, 7078 (1987)

34. Irwin, M.C.: Smooth dynamical systems. New York: Academic Press 1980

35. Löwen, H., Beier, T.: Phys. Rev. B (in press)

36. See e.g.: Dietrich, S.: In: Phase transitions and critical phenomena. Domb, C., Lebowitz, J. (eds.), Vol. 12, p. 1. London: Academic Press 1988

37. McRae, E.G., Malic, R.A.: Phys. Rev. Lett. 58, 1437 (1987)

H. Löwen, T. Beier, H. Wagner

Sektion Physik der Universität München

Theresienstrasse 37

D-8000 München 2

Federal Republic of Germany 